\title{
IR of Iran National Mobilization against COVID-19 Epidemic
}

\author{
Alireza Raeisi, MD'; Jafar Sadegh Tabrizi, MD, PhD²,3*; Mohammad Mehdi Gouya, MD ${ }^{4}$ \\ ${ }^{1}$ School of Medicine, Shiraz University of Medical sciences, Shiraz, Iran; Health Deputy, Ministry of Health and Medical Education, Tehran, \\ Iran \\ ${ }^{2}$ Health Services Management Research Center, School of Health Management and Medical Informatics, Tabriz University of Medical sciences, \\ Tabriz, Iran \\ ${ }^{3}$ Primary Health care network Management Center, Ministry of Health and Medical Education, Tehran, Iran \\ ${ }^{4}$ Iran University of Medical sciences, Tehran , Iran; Center for Disease Control, Ministry of Health and Medical Education, Tehran, Iran
}

$\mathrm{T}$ 2019 novel coronavirus (SARS-CoV-2), which was first reported in December 2019 in Wuhan, China has since spread across the world. Iran has one of the fastest doubling rates for SARS-CoV-2 infection and hence a high rate of morbidity and mortality for CIVD19. The Health Care Networks of the Islamic Republic of Iran (HCNIR $)^{1,2}$ played a major role for control of epidemic in Iran.

The Health Care Networks of the Islamic Republic of Iran were launched in 1985 and extended to the entire nation in $1990 .{ }^{1}$ The Networks mostly covered rural areas while urban areas lacked complete coverage. Following the implementation of the Health Transformation Plan (HTP) in 2015, the Network witnessed an improvement in status in urban and suburban areas, yielding acceptable coverage to all urban and rural populations. ${ }^{3}$ Currently, primary health care is accessible to all people within a 10-min commute on public transportation.

In rural areas, the Network comprises health houses (HHs) and rural comprehensive health centers (RCHCs). Each RCHC covers, on the average, five health houses. Through rural community-based health workers (CHWs) known as Behvarz, each health house covers an average population of 1000 individuals, providing active preventive care, health promotion, screening, and simple symptomatic treatment services for predefined conditions as well as referrals to RCHCs (in cases requiring medical care) and district hospitals (in emergency cases). In rural areas, more than 17800 health houses employing 31000 Behvarzes, as well as about 2794 RHCs employing 6642 family physicians and 5852 rural midwives are offering their services to the 28 -million-person rural population. Thus, $98 \%$ of the rural population is actively covered by primary health care (Figure 1).

Health posts (HPs) constitute the first line of health care provision in urban areas, offering services (similar to those of rural health houses) to urban and suburban populations through urban community-based health workers (Moragheb-e-salamat). Each urban CHW is responsible for providing active primary care to an average of 2500 individuals. Each Urban Comprehensive Health Center (UCHC) covers an average of three health posts. Throughout the urban areas of the nation, there are 5343 HPs and 2723 UCHCs, employing more than 24000 urban

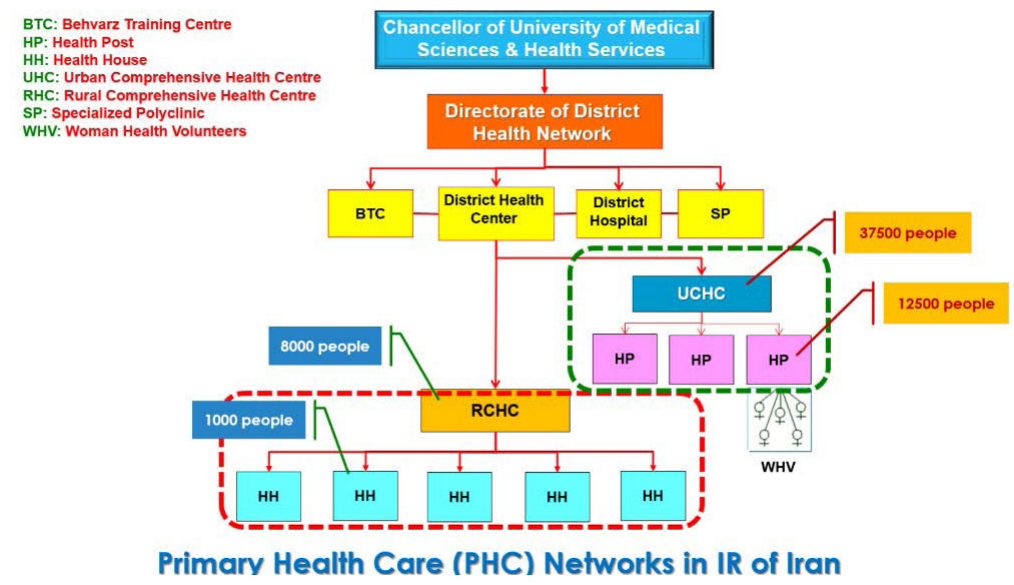

Figure 1. Primary Health Care (PHC) Networks in I.R. of Iran.

*Corresponding Author: Jafar Sadegh Tabrizi, MD, PhD; Health Services Management Research Center, School of Health Management and Medical Informatics, Tabriz University of Medical sciences, Tabriz, Iran. Email: js.tabrizi@gmail.com 
CHWs, 3987 physicians, and 12032 other care providers (dentists, nurses, midwives, and professionals addressing occupational and environmental health, nutrition, mental health, etc) to offer their services to the urban population of 54 million individuals. Currently, $93 \%$ of the urban population is covered by primary health care.

RCHCs and UCHCs refer patients requiring more specialized care to specialized clinics or district hospitals in the townships. The entirety of the mentioned urban and rural health care providers in a township constitute the Health Network of the township, functioning under the supervision of the University of Medical Sciences of its province. Since 2016, electronic health records have been implemented in order to register and manage the services offered in primary health care units. This project was completed in May 2019 and more than 95\% of the population now have an electronic health record. Currently, all primary health services provided to individuals of all ages are documented, enabling follow-up and evaluation.

Following the spread of COVID-19 in China, the Deputy for Health at the Iranian Ministry of Health and Medical Education (MOHME) translated and compiled guidelines for COVID-19 prevention and treatment, according to the recommendations of the World Health Organization (WHO). In addition, in-person education was provided to improve the knowledge and skills of Behvarzes, urban CHWs, midwives, nurses, physicians, and other professional employed by the health care network throughout the country in preparation for the spread of the coronavirus. Following the educational course, the diagnostic and treatment procedures for COVID-19 were uploaded to the electronic health system. Alongside in-person education, the protocols for services and their registry in the system were sent via morning welcome messages on different platforms to all users to prepare them for practice.

Following the spread of COVID-19 in the I.R. of Iran, alongside the efforts started by the treatment system (specialized and sub-specialized clinics and hospitals) and the laboratory network, the primary health network launched its active education regarding personal, familial, and social prevention to target the entire rural and urban population of the country. Five days after the first patient was diagnosed with COVID-19 in the city of Qom, the MOHME designed and launched its National Mobilization Plan against COVID-19 using the motto \#We-WillOVERCOME-Corona. This plan relies on a collaboration between the Ministry's Deputy for Health and Basij forces, health ambassadors, neighborhood health volunteers, and volunteers from non-governmental organizations (NGOs), students, etc. The plan consists of six major segments.

Segment 1. Hotlines 190 and 4030 with over 10000 lines to respond to questions and provide general and specialized advice, including recommendations on nutrition, mental health and COVID-19 selected and referral centers.
Segment 2. Launching 1000 selected comprehensive health centers functioning 16 and 24 hours per day, seven days of the week, to provide specific COVID-19 services according to the national protocol. Over a period of one week, these centers were equipped with pulse oximeters and laser thermometers for screening purposes. The Deputy for Health plans to launch sampling units to screen for coronavirus in these centers; however, it is regrettable that sanctions enforced by the United States and lack of diagnostic kits have undermined these efforts.

Segment 3. The website salamat.gov.ir for screening based on data entry. This website is linked to the electronic health records. Throughout the country, people are able to access this website on entering their national identification number and birth of date, answer simple questions for COVID-19 evaluation, and receive relevant advice according to the latest guidelines of the MOHME. This will impede the spread of coronavirus in crowded health centers by preventing many people from unnecessarily referring to health centers in person.

Once logged in to the website, the user is inquired about symptoms (fever, chills, dry cough, sore throat, difficulty breathing) as well as the presence of individuals with suspected COVID-19 symptoms in their family. Positive cases are provided with recommendations about personal and familial protection and referred to the nearest selected comprehensive health center. Simultaneously, an urban $\mathrm{CHW}$ (in cities) or Behvarz (in villages) receives a text message on the phone and is put in charge of subsequent follow-up for the suspected patient and contact tracing. The responsible urban CHW or Behvarz immediately contacts the suspected patient on the free-of-charge 4030 line to provide necessary counsel and follow-up. Whether the patient is staying at home or admitted to a hospital, a team is dispatched to their domicile or workplace for contact tracing and evaluation (Further details in segment 5 below). These measures are intended to establish personal hygiene, home quarantine, and disruption of virus transmission.

Segment 4. Phone screening of households covered by Behvarzes and urban $\mathrm{CHW}$ s based on electronic health records. Behvarzes in villages and urban $\mathrm{CHW}$ in cities contact household heads on the 4030 line, prioritizing the high-risk population (those with chronic diseases such as diabetes and hypertension, obesity, and immunodeficiency, the elderly, those with malignancy, those undergoing chemotherapy, and pregnant women) to inquire about COVID-19 symptoms (fever, chills, dry cough, sore throat, difficulty breathing) in their family members. The information is registered in electronic health records. In case of finding suspected individuals, personal protection measures are instructed and the person is referred to the nearest selected comprehensive health center.

In the selected comprehensive health center, the 
referrals are evaluated according to the national protocol for COVID-19; those with symptoms and an oxygen saturation below 93\% are referred to a COVID-19 referral hospital (which is going to have corona virus test in the next 7 days as well). For those who do not need referral, outpatient treatment is administered in the form of hydroxychloroquine for five days and daily follow-up by the Behvarz or urban CHW on the phone or will refer to the quarantine centers to have pre-hospital care under medical staff supervision. In addition, the companions of the patient are educated about home quarantine, symptom exacerbation, and personal protection. Patient who have only one symptom are advised to stay at home and follow health recommendations.

As of March 22, 2020, the implementation of the third and fourth segments has resulted in the screening of more than 37 million individuals, including 29767000 individuals screened on the phone by Behvarzes and urban CHWs, and about 7275000 individuals who self-evaluated on salamat.gov.ir. Figure 2 depicts the screened population for each province.

Out of 37 million people screened, a total of $1.45 \%$ (approximately 417000 individuals) only had one symptom, and about $0.2 \%$ (66960 individuals) were referred to selected centers (Figure 3).

Among the individuals referred to selected comprehensive health centers, about $26 \%$ required care at home, $0.65 \%$ received outpatient hydroxychloroquine treatment for five days according to the national protocol (documented by physicians in electronic health records), and $4.37 \%$ were referred to COVID-19 referral hospitals (Figure 4). The Behvarzes and urban CHWs are responsible for following those receiving outpatient treatment over a period of six days, registering data regarding symptom exacerbation, recovery, or possible impaired consciousness in the system, and if necessary, referring the patient to selected centers or referral hospitals (in emergency cases). Out of 9529 patients (4.37\%) referred to hospitals, 33.67\% (3208 patients) were admitted; the rest were discharged with recommendation

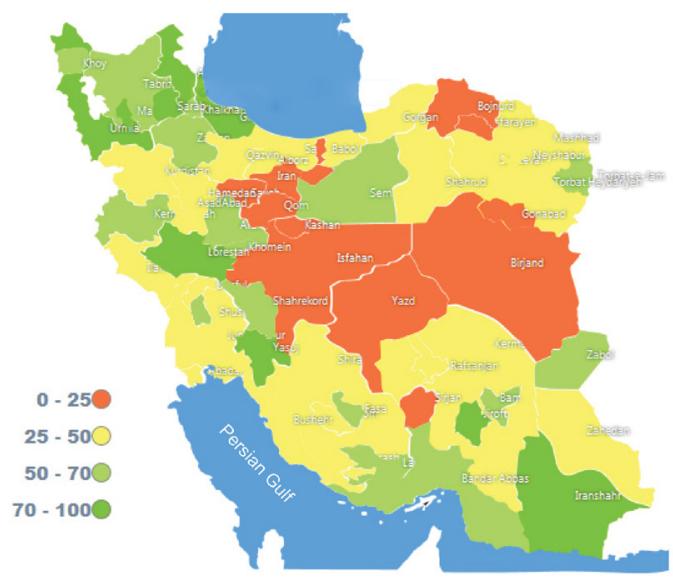

Figure 2. Percentage of Screened Population for Each Province. of staying at home and following personal and familial health advices, referring to the quarantine centers or with outpatient treatment (hydroxychloroquine) and instructions on home quarantine and care at home.

\section{Segment 5. National Mobilization Against COVID-19} teams.

The composition of executive teams for coronavirus prevention and control is summarized in the following:

1. Care teams (2-3 persons: Behvarzes/urban CHWs and Basij forces);

2. Environmental sanitation intervention teams (3-5 persons: occupational/environmental health professionals, Basij forces, other volunteers);

3. Organizations and companies Basij teams (2-4 persons: Basij, NGOs, and volunteer employees);

4. City entrance checkpoint teams (4 persons: The Red Crescent, Police, Basij forces, and emergency medicine).

For the purpose of identifying home visit teams, once a household is selected by the Behvarz/urban CHW, text messages containing an identification code are simultaneously delivered to the household head and team members. When the care team arrives at the designated home, their identity is confirmed by matching the codes sent to the household head and team members. Prior to launching this program, an extensive campaign was started about the procedure, symbolic presentation of team members and their activities on the national television,

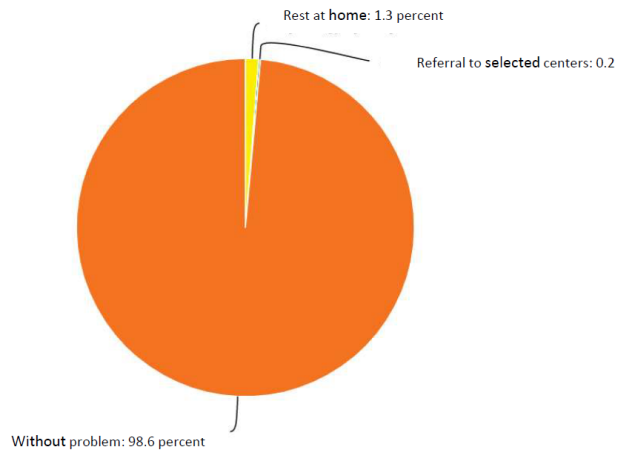

Figure 3. The Population Phone Screening by Behvarzes and Urban CHWs.

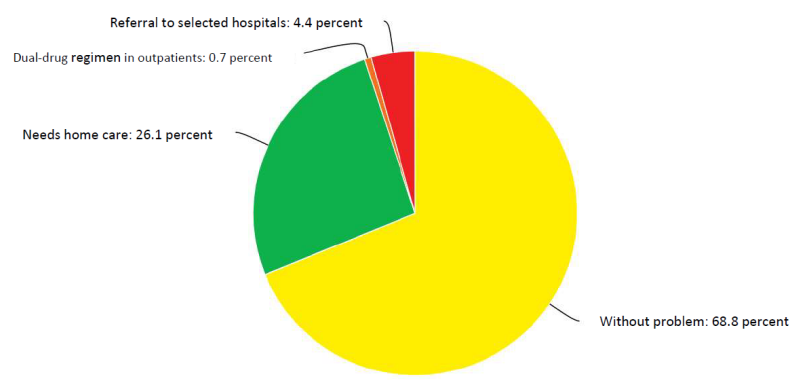

Figure 4. The Population Screened and Treated at Selected Comprehensive Health Centers. 
local television stations, and other mass media to inform the population about the project.

All staff in these teams are supplied with protective equipment of measures, such as goggles, three-ply face masks, and gloves, for each home visit according to the guidelines of the MOHME. The educational materials intended for National Mobilization Against COVID-19 teams are accessible to all members at http://iec.behdasht. gov.ir/.

\section{Care Teams}

The care teams are responsible for education, screening, follow-up and disinfection with their members selected from among Behvarzes/urban CHWs and Basij forces. They trace the contacts of individuals exposed to the target group, provide education about disease prevention and control, follow on patients treated at home, educate and enable households regarding isolation and care, establish environmental protective measures in houses, complete the follow-up forms and register them in the electronic health record system, and refer suspected cases to selected health centers.

\section{Environmental Sanitation Intervention Teams}

These teams are responsible for education, supervision, control, and monitoring the implementation of MOHME protocols in public places and food supply and distribution centers. The team members include occupational/ environmental health professionals, Basij forces, and volunteers.

Equipped with personal protection equipment, these teams visit food supply and distribution centers, public places, and working places to ensure compliance with MOHME protocols for COVID-19 prevention and control. The team is responsible for disinfection of public spaces, vigorous supervision on food supply and distribution centers, preventing crowds, guiding transportation control in contaminated areas, mapping of all public places, and encouraging the cooperation of proprietors.

\section{Organizations and Companies Basij Teams}

These teams are responsible for educating employees, controlling entry and exit points, and disinfection of public and restricted spaces in workplaces. For each organization or company, the team is composed of its own trained Basij forces or volunteer employees. The teams are equipped act with personal protection equipment according to the MOHME guidelines.

The teams use laser thermometers to evaluate employees and clients at the entry points. Continuous disinfection is also performed in accordance with the MOHME guidelines.

\section{City Entrance Checkpoint Teams}

Under supervision of the I.R. of Iran Red Crescent Society, these teams are located at city entry and exit points for the purpose of education and evaluation. Team members include 4 persons from the Red Crescent, Basij forces, the Police, and emergency medicine.

Equipped with personal protection equipment according to the MOHME protocols, these teams assess body temperature, educate, and evaluate travelers at checkpoints and guide the suspected cases towards designated quarantine units.

Segment 6. National Campaign for COVID-19 Public Awareness and Information

Aiming to improve knowledge regarding COVID-19, the Iranian Ministry of Health and Medical Education has launched a National Campaign for COVID-19 Information since February 23, 2020. This $360^{\circ}$ campaign employs the capabilities of the national television, cyberspace, out-ofhome advertising, and information kiosks throughout cities based on the covered population and with collaborations from the Deputies of the MOHME, all universities/ schools of medical sciences throughout the nation, mayoral offices, the Basij organization, and other collaborating organizations.

The MOHME website at http://iec.behdasht.gov.ir includes all educational materials developed for urban public spaces, communication on the media, and public education.

\section{Authors' Contribution}

AR conceptualized and designed the study, edited and critically reviewed manuscript, and collaborated in quality control. JST collaborated in data processing, designed the study and wrote the manuscript. MMG collaborated in data collection, edited and critically reviewed manuscript. All authors read and approved the final manuscript.

\section{Conflict of Interest Disclosures}

None.

\section{Ethical Statement}

Not applicable.

\section{References}

1. Shadpour, K. Primary health care networks in the Islamic Republic of Iran. EMHJ - Eastern Mediterranean Health Journal. 2000;6(4):822-825.

2. Moshiri E, Rashidian A, Arab M, Khosravi A. Using an analytical framework to explain the formation of primary health cCare in rural Iran in the 1980s. Arch Iran Med. 2016;19(1):16-22

3. Sajadi HS, Majdzadeh R. From primary health care to universal health coverage in the Islamic Republic of Iran: a journey of four decades. Arch Iran Med. 2019;22(5):262-8. 